\title{
Blockchains, Formulary Evaluations and Health Technology Assessment
}

Paul C Langley, PhD ${ }^{1}$; Robert E Martin, MS RPh ${ }^{2}$

${ }^{1}$ Adjunct Professor, College of Pharmacy, University of Minnesota

${ }^{2}$ Folsom CA

\begin{abstract}
Blockchains have the potential, if appropriately implemented and managed, of contributing to a fundamental change in the application of techniques of health technology assessment in formulary decision making. Rather than continuing to rely on claims made by manufacturers that are non-evaluable, the introduction of blockchains as adjunct to the electronic medical record, meet an unmet medical need in enabling a platform for the real time assessment of claims. Rather than focusing the case for their product on lifetime cost-per-QALY models, which have no chance of ever being validated, the blockchain platform offers a low cost opportunity for claims evaluation. Manufacturers would be required to abandon the construction of imaginary cost-per-QALY worlds to support claims for pricing and formulary trier position, in favor of claims that can be evaluated and reported back to formulary committees in a short yet meaningful time horizon. Manufacturers would present a claims assessment protocol as part of the formulary submission package. If agreed with the formulary committee, the protocol would be implemented and managed through the patient blockchain membership. Claims would be monitored and evaluated in real time with a final report to the formulary committee in a matter of months. Lifetime imaginary claims for cost-per-QALY, set alongside willingness-to-pay thresholds, would be a thing of the past. Pricing decisions and formulary placement would reflect a robust evidence base and not just extrapolations from pivotal clinical trials.
\end{abstract}

Keywords: blockchain technology, blockchains in health, barriers to blockchain implementation, health data property rights

\section{INTRODUCTION}

Establishing a robust evidence base to support, not only formulary decision making for new products and devices, but also to support ongoing disease and therapeutic class reviews is essential for effective health system management ${ }^{1} 2$. Unfortunately, when new products and devices are proposed for formulary listing the evidence base is typically far from robust. Apart from the limitations imposed on claims for external validity that characterize phase 3 pivotal trials, claims presented are all too often extrapolated from trials with a limited time frame. Attendant claims for cost-effectiveness are all too often supported by modeled frameworks that defy evaluation and replication. In previous commentaries in INNOVATIONS in Pharmacy the case has been made that the standards recommended for formulary submission reflect a willingness to extrapolate from limited clinical data to lifetime clinical and cost-outcomes models ${ }^{3}$ The key criticism is that , irrespective of claims that the assumptions are 'realistic' constructing imaginary modeled worlds fail to meet the standards of normal science in generating credible, evaluable and replicable claims ${ }^{4}$. This lack of scientific merit is exemplified in the US by the standards for formulary submissions advocated by groups such as the Academy of

\section{Corresponding author: Paul C Langley, PhD}

Adjunct Professor

College of Pharmacy University of Minnesota

Director, Maimon Research LLC

5061 North Apache Hills Trail, Tucson, AZ 85750

Email: langley@maimonresearch.com
Managed Care Pharmacy (AMCP) and the International Society for Pharmacoeconomics and Outcomes Research (ISPOR) as well as by self-appointed arbiters of cost-effectiveness claims ${ }^{5}$ ${ }^{6}$. The last point is best exemplified by the reports prepared by the Institute for Clinical and Economic Review (ICER) ${ }^{7}$. The ICER is wedded to the lifetime cost-per-QALY methodology that generates claims that are impossible to verify (by definition). The result, as detailed below, is that manufacturers respond by producing competing models which are claimed to be more realistic in their choice of lifetime modeled assumptions. The net result is a number of competing models that serve no other purpose than to 'muddy' the waters. Or, to put somewhat differently, a situation where various black box models compete for attention.

The purpose of this commentary is to explore the potential implications for formulary evaluations of a health system adopting a blockchain platform that sits alongside the electronic medical record; a potential role that allows us to put to one side the construction of imaginary worlds to support formulary advocacy. Rather, we have the opportunity to move towards a formulary evaluation paradigm that rejects the standard approach to submissions in favor of one that emphasizes the need for competing drug and device claims that are realistic, evaluable and replicable in treating environments. A process that allows formulary committees to receive timely evaluations of claims for target patient populations within the approved indication that can be integrated in pricing and tier position decisions. 


\section{Blockchains, Property Rights and Security}

The technical merits of blockchains are now, after some ten years since their first introduction to support cryptocurrency exchanges, well established. They offer a secure, encrypted data environment which overcomes, from the patient's perspective, concerns with the security of their personal health and medical records. Subject to agreement on property rights to medical records and the transfer of records to the blockchain, the prospect opens up for the patient, subject to the appropriate permissions and 'smart contracts', to monetize their data via tokenization. Additionally, the patient also has the opportunity to value add to their record by participating in studies that support assessments of therapy responses.

Two previous have explored the range of activities that blockchain platforms could support in health technology assessment including formulary evaluations ${ }^{8} 9$.

It is this last prospect, supporting studies that evaluate claims for competing products and devices, as well as new products, that offers formulary committees a more robust evidence base for pricing and tier positioning.

Even so, it should be cautioned that the ability to support claims assessments, as detailed in our previous commentary, presupposes the role of a managed market coordinator. The blockchain owner, or a sub-contractor, would have the task of acting as a market intermediary between the health system formulary, the manufacturer, providers and patients. This is a necessary condition for the blockchain platform to realize its potential with the owner as agent for the patients subscribing to the blockchain. A primary task for the blockchain owner, as agent, would be to identify target patient population within the platform and to solicit their agreement for monetized study participation.

\section{Blockchain Incentives}

Who benefits from the blockchain platform? If we accept the proposition that the blockchain is 'free' to the patient who is encouraged to participate through secure encryption and prospective monetization of their health data, the issue is then one of incentivizing the other participants: the health system and the provider.

If we focus on the role of the blockchain platform as a necessary element in formulary evaluations, then the potential benefits to the health system in subscribing to the blockchain (e.g., for profit HMO) lies in the ability of the health system to generate, in real-time, a platform for evaluating claims for competing products for target patient groups. These would have the potential for monitoring patients' response and switching interventions leading to improved outcomes in target populations and reduced resource misallocation. A robust claims assessment potential would put health systems in the pole position in negotiating prices. Rather than rely on a modeled claim, there would be real world evidence (RWE) to judge the merits of competing products. This feedback could also be counter to the practice of manufacturers announcing annual or semi-annual price increases where there is no clinical justification. Indeed, a blockchain monitoring system would enable closer targeting of patients who are expected to benefit, supported by programs to improve adherence and reduce discontinuation.

The option of developing blockchain platforms in disease areas also offers a secure base for risk contracting with manufacturers. In the terms of both supporting platform claims assessment and utilizing the platform to support a risk sharing contract, the manufacturer (as detailed below) would be expected to meet all costs.

The provider or physician practice plays a pivotal role in both the recruitment of patients to the blockchain, as well as, supporting value added evaluations of claims for new and competing products and devices. Options for provider incentives would include: (i) tokenization for study participation; (ii) enhanced practice revenue through participating in a study; (iii) as well as support through ongoing data transfers to the blockchain. Potential revenue sources are from meeting CMS quality metric initiatives as the Federal government focuses on outcomes rather than process measures, and the possibility of capturing billable CPT codes from practice improved data collection (e.g., patient reported outcomes) and transfer of these data to the blockchain.

\section{Formulary Submission Standards}

Over the past almost 20 years the AMCP Format for Formulary Submissions has become the de facto standard for formulary submission in both public and commercial environments. This is unfortunate from the claims assessment perspective. While the format asks for a summary of the clinical attributes of the particular product, together with an assessment of its relative merits vis à vis comparator products, consideration is not given to claims assessment and replication in target populations or how the claims might be evaluated. This is a major oversight given the doubts that have been raised over the past decade and more that RCT based claims, even when the same protocol is used, have been impossible to replicate.

Apart from the absence of recommendations for claims replication, the format recommends that, in making a claim for cost-effectiveness, manufacturers should present a model that tracks the natural course of a disease. The model should express outcomes in terms of cost-per-quality adjusted life year (QALYs) with modeled outcomes set against willingness-to-pay thresholds. Given uncertainties in model structure and model parameters a sensitivity analysis is recommended, with estimates of the probability of being cost-effective in terms of the unit price of the product. 
Obviously, modeling over the lifetime of a target patient cohort - whose characteristics may be selected to enhance model claims - with a price that may similarly be chosen to support claims for cost-effectiveness and willingness-to-pay, is hardly a basis for effective formulary decision making. It is perhaps not surprising that formulary committees are suspicious of modeled claims, particularly when there is no chance of the claim ever being evaluated. The fact is that any modeled claim for cost-effectiveness to meet willingness-to-pay thresholds can be constructed. A price can be selected by the manufacturer simply to obtain formulary admission and, within a few months, revised to meet pricing strategy objectives. While this may seem an unduly cynical perspective in light of the resources devoted to modeling and the thousands of papers published over the past 30 or so years, the fact remains that in the absence of an acceptance of the standards of normal science reverse engineering is always possible.

Unfortunately, the popularity of lifetime modeled claims seems impervious to the criticism that constructing imaginary claims fails to meet the standards of normal science. In part this reflects the fact that assessment agencies such as the National Institute for Health and Care Excellence (NICE) in the UK, as agent for the National Health Service (NHS) in England, mandate imaginary cost-per-QALY models to emulate the natural course of a disease ${ }^{10}$. Indeed, the latest Canadian guideline for formulary submissions is quite clear that building imaginary worlds is for 'information only' ${ }^{11}$. They are not interested in evaluating claims: Economic evaluations are designed to inform decisions. As such, they are distinct from conventional research activities, which are designed to test hypotheses. It is a moot point as to how the construction of imaginary worlds which may extend over decades (if intended to capture the natural course of a disease) are expected to inform decisions when, presumably, competing imaginary claims may also be submitted. In the absence of a commitment to evaluate claims through hypothesis testing it is difficult to see why formulary committees should take modeled claims for cost-effectiveness seriously.

\section{The Blockchain Alternative}

Rather than basing decisions on non-evaluable claims generated by imaginary worlds, the blockchain platform offers a robust evidentiary alternative. An alternative that is consistent with the standards of normal science for evaluation and replication of clinical and, in the short-term, costeffectiveness claims. The presence of a blockchain platform obviates the need to construct imaginary worlds. Rather than a manufacturer pleading with a formulary committee and even offering discounts to justify their model in contrast to competing models, the blockchain platform can support evaluation protocols.
The requirement for manufacturers to support and underwrite evaluation protocols is central to the recently released Minnesota guideline for formulary submissions ${ }^{2}$. Where a submission is prepared for a new product or to support ongoing disease area and therapeutic class reviews the manufacturer is asked to present as part of the submission a protocol describing how the clinical and cost-effectiveness claims are to be evaluated. Lifetime modeled claims are rejected; claims should be able to be evaluated in a short timeframe and the results reported back to the formulary committee. Until the results of the claims assessment protocol are available the product is granted provisional pricing and tier placement.

With the blockchain property rights vested in the patient, implementing a claims assessment protocol will require the manufacturer to enter into monetized, smart contracts with target patient populations. Depending upon the data elements that are required to be captured, further agreement may have to be sought with providers to capture office assessed clinical endpoints.

A key point to note is that the protocol is intended not only to evaluate but provide a forum to replicate claims generated by pivotal randomized clinical trials (RCTs), as closely as possible. Rather than taking RCT claims at face value, which is the case in the construction of imaginary worlds, requiring a claims protocol addresses the issue of non-replication. There is now abundant evidence that a significant proportion of RCT claims, in some instances in excess of $50 \%$, cannot be replicated when the identical protocols are applied to target patient populations - including attempted replication by the original investigators. Failure to replicate claims from pivotal phase 3 trials undercuts any modeling framework and consequent cost-effectiveness claims.

At the same time, unlike the typical design of phase 3 pivotal RCTs, the Minnesota guidelines ask that the protocol takes explicit account of comparator therapies. Rather than claims for new products against placebo, which are the basis for marketing approval, the formulary committee can insist on a claims assessment against the standard of care. The protocol does not exclude the possibility of a placebo arm if that is considered appropriate by the formulary committee.

Consider, as a case in point, claims expressed in both clinical and quality of life terms. Adopting a blockchain platform, a protocol evaluation would allow clinical response and endpoints (at timed intervals) to be reported, but also resource utilization in a real world treating environment and patient reported quality of life outcomes (PROs). The protocol could not only replicate the PROs found in the original trial but could add further PROs to assess, for example, patient perceptions of response to therapy. As well, a protocol could address the fact that in RCTs secondary as opposed to primary outcomes are 
typically underpowered. Rather than restricting the protocol to a limited sample size, noting that a blockchain protocol allows randomization to treatment arms, the target population can be expanded to allow the appropriate power for 'secondary' outcomes. It is worth noting that modeled claims for cost effectiveness often utilize underpowered endpoints to justify modeling assumptions.

\section{Compliance and Response}

The requirement for a claims assessment protocol that attempts to imitate the RCT parameters also has the potential to address the question of compliance. It is now taken as axiomatic that the majority of patients initiated to a range of therapies discontinue these within 12 to 18 months. These patterns stand in contrast to compliance behavior in RCTs where, apart from the short-term nature of many of the trials where discontinuation will be minimal in any case, efforts are made to ensure compliance. Unfortunately, one of the presumably unintended consequences of attempting to model by extrapolating from a short-term RCT, is that imaginary world model builders build in long-term assumptions regarding compliance which overstate observed behavior. A number of ICER reports illustrate this shortcoming ${ }^{12} 13$.

Application of a protocol to blockchain patients, in a protocol that focuses on response in a real world environment (RWE) allows for accountability not only of compliance behavior, including both adherence and discontinuation, but also the reasons for non-compliance. A low rate of compliance, whether due to poor response, adverse events or the patient simply seeing no reason to continue therapy, gives the formulary committee a powerful argument in pricing negotiations and tier placement. If the analysis of response suggests that certain characteristics are associated with poor compliance, the committee may recommend interventions to restrict losses due to non-response. This may not appeal to manufacturers, where the focus is on sales rather than compliance. If a sales target can be achieved by new patients being introduced to therapy matching or exceeding those dropping out, then there is little incentive to probe compliance behavior.

\section{Comorbidities and Older Populations}

A criticism that is often made of the typical RCT is that patients with comorbidities are excluded from the protocol. Excluding comorbidities is of particular concern in older populations; a group typically excluded from RCT designs. The ability to utilize a blockchain platform allows formulary committee to consider, in a protocol design whether to include comorbidities in patient recruitment together with older populations. The fact that a protocol has been agreed with the primary purpose of replicating RCT results does not exclude developing and requiring manufacturers to support other protocols in older populations and those with comorbidities. RWE protocols may be expected to qualify response claims, while providing external validity.

The importance of being able to develop protocols in a blockchain environment should not be understated. Rather than attempting to argue, from a limited or absent evidence base, for the applicability of RCT claims based on pivotal trials to more complex and older populations, formulary committees and other health decision makers are in the position of being able to require manufacturers to undertake studies at a relatively low cost and in a meaningful time frame.

\section{The Long Term}

Once a protocol is in place for a target population there is no reason why patients enrolled in the protocol could not be followed in the long-term. A manufacturer may agree, if the focus of the indication and claims is on the long-term why the protocol embedded in the blockchain could not become, in effect, a real-time registry.

\section{Implications for Cost-Effectiveness Claims}

Adoption by health care systems of blockchain platforms promise to transform the present reliance on non-evaluable modeled clinical and cost-effectiveness claims. Basing formulary decisions on submissions by manufacturers that put the standards of normal science to one side in favor of nonevaluable 'for information' claims will be a thing of the past. Manufacturers will have to focus on the role of hypothesis testing in putting forward clinical and cost-effectiveness claims. Lifetime or long-term cost-per-QALY models will not be accepted. As noted above, the possibility of implementing protocols to assess claims will eliminate the need to focus on models if these models generate non-evaluable claims.

A further casualty of the blockchain platform will be the plethora of statistical and mathematical techniques that have been developed to support lifetime models. Rather than imaginary constructs whose claim for relevance is based on the 'realism' of their assumptions, this instrumentalist approach can be rejected in favor of an evidence based paradigm that allows patients to be tracked in real time over their course of treatment, generating RWE.

For a health technology perspective, the blockchain offers a platform that moves away from the limited endpoints that characterize pivotal RCT protocols to an environment where outcomes capturing a range of PROs can be accommodated. If quality of life is an endpoint, then the blockchain facilitator can enter into contracts with patients in target groups to evaluate cost-per-QALY claims. If functional status is an endpoint, this can also be captured from the perspective of the provider. Monitoring functional status, for example, can provide the basis for assessing whether a minimal important clinical improvement has occurred between office visits as well as the 
patient's perspective on this change. The net result is the prospect of a quantum change in the evidence base to support health care.

\section{Conclusions}

The potential for blockchains to support health technology assessments promises a paradigmatic shift in the evaluation of competing pharmaceutical and device claims. Rather than relying on modeled claims, defended on the basis of the realism of their structure and assumptions, health care decision makers can address directly the RWE credibility or otherwise of claims. Evidence replaces modeled information.

Conflicts of Interest: No conflicts were declared by either PCL or REM 


\section{References}

${ }^{1}$ Langley PC. Nullius in Verba: Version 2.0 of the University of Minnesota, School of Social and Administrative Pharmacy Program, Proposed Guidelines for Formulary Evaluation. Inov Pharm. 2016;7(4): No 16

${ }^{2}$ Langley PC. Guidelines for Formulary Evaluation [Proposed]. Program in Social and Administrative Pharmacy. College of Pharmacy. University of Minnesota. Version 2.0. December 2016. http://www.pharmacy.umn.edu/departments/pharmaceutical-care-health-systems/departmentprograms-and-centers

3 INNOVATIONS in Pharmacy https://pubs.lib.umn.edu/index.php/innovations/section/view/formularyevaluations

${ }^{4}$ Langley PC. Validation of modeled pharmacoeconomic claims in formulary submissions. J Med Econ. 2015;18(12):993-99

${ }^{5}$ Langley PC. Modeling imaginary worlds: Version 4 of the AMCP Format for Formulary Submissions. Inov Pharm. 2016;7(2): No. 11

${ }^{6}$ Langley PC. Supporting formulary decisions: The discovery of new facts or constructed evidence? Inov Pharm. 2016;7(2): No.15.

${ }^{7}$ Langley PC. Resolving Lingering Problems or Continued Support for Pseudoscience? The ICER Value Assessment Update. Inov Pharm. 2017;8(4): No 7

${ }^{8}$ Langley PC. Information or evidence: Abandoning imaginary worlds for blockchains in health technology assessment. Inov Pharm. $2018 ; 9(3)$

${ }^{9}$ Langley PC, Martin RE. Blockchains , Property Rights and health Technology Assessment in the Pharmaceutical and Device(s) Industries. Inov Pharm. 2018;9(4): No. 1

10 Langley PC. Sunlit uplands: the genius of the NICE reference case. Inov Pharm. 2016;7(2): No.12.

${ }^{11}$ Langley PC. True North: Building Imaginary Worlds with the Revised Canadian (CADTH) Guidelines for Health Technology Assessment. Inov Pharm. 2017;8(2): No. 9.

12 Langley PC. Cost-Effectiveness and Formulary Evaluation: Imaginary Worlds and Entresto Claims in Heart Failure. Inov Pharm. 2016;7(3): No. 6

${ }^{13}$ Langley PC. Multiple Sclerosis and the Comparative Value Disease Modifying Therapy Report of the Institute for Clinical and Economic Review (ICER). Inov Pharm. 2017;8(1): No. 12 